\title{
Tempo despendido no sistema de assistência de enfermagem após implementação de sistema padronizado de linguagem
}

\author{
TIME SPENT ON THE NURSING ASSISTANCE SYSTEM AFTER \\ THE IMPLEMENTATION OF A STANDARD LANGUAGE SYSTEM \\ TEMPO UTILIZADO EN EL SISTEMA DE ASISTENCIA DE ENFERMERÍA
DESPUÉS DE LA IMPLEMENTACIÓN DEL SISTEMA DEL LENGUAJE PATRÓN
}

Priscila Oliveira Rezende', Raquel Rapone Gaizinski ${ }^{2}$

\section{RESUMO}

Este estudo, exploratório-descritivo prospectivo, objetivou analisar o efeito da mudança do modelo do Sistema de Assistência de Enfermagem (SAE), com a introdução de classificação de diagnósticos de enfermagem, no tempo despendido pela enfermeira na realização desse Sistema, identificando o tempo estimado e o medido (cronometrado). A amostra foi constituída por 42 enfermeiras, em média, com 11 anos de experiência profissional, de diferentes unidades do HU-USP, sendo observadas, cinco vezes, em média, executando as fases do SAE. Os dados foram coletados no período de agosto/2005 a fevereiro/2006, após a introdução e sedimentação da classificação de diagnóstico de enfermagem. Os resultados evidenciaram que o tempo estimado pelas enfermeiras foi significativamente maior ao tempo medido e que o tempo despendido com a avaliação do paciente foi, em média, maior na admissão do paciente em relação ao seu seguimento.

\section{DESCRITORES}

Processos de enfermagem.

Recursos humanos de enfermagem.

Diagnóstico de enfermagem.

Carga de trabalho.

\begin{abstract}
This prospective, exploratory-descriptive study was aimed at analyzing the results of the change of model of the Nursing Assistance System (SAE), with the introduction of a nursing diagnosis classification, on the time spent by the nurse in the realization of this system, identifying the estimated time and the measured time. The study was based on a sample of 42 nurses from different units of the University of São Paulo's Hospital Universitário, with in average 11 years of professional experience, who were observed in average 5 times while carrying out the phases of the SAE. The data were collected from August, 2005 to February, 2006, after the introduction and consolidation of the nursing diagnosis classification. The results showed that the time estimated by the nurses was significantly longer than the time actually measured; and that the time spent for patient evaluation was, on average, longer during his/her admission compared to the follow-up.
\end{abstract}

\section{KEY WORDS}

Nursing process.

Nursing staff.

Nursing diagnosis.

Workload.

\section{RESUMEN}

En este estudio, exploratorio-descriptivo prospectivo, se tuvo como objetivo analizar el efecto de cambio del modelo del Sistema de Asistencia de Enfermería (SAE), con la introducción de la clasificación de diagnósticos de enfermería, en el tiempo utilizado por la enfermera en la realización de ese Sistema, identificando el tiempo estimado y el medido (cronometrado). La muestra estuvo conformada por 42 enfermeras, en promedio, con 11 años de experiencia profesional, de diferentes unidades del HU-USP, siendo observadas, cinco veces, en promedio, cuando ejecutaban las fases del SAE. Los datos fueron recolectados en el período de agosto/2005 a febrero/ 2006, después de la introducción y sedimentación de la clasificación del diagnóstico de enfermería. Los resultados permitieron evidenciar que el tiempo estimado por las enfermeras fue significativamente mayor al tempo medido y que el tiempo gastado en la evaluación del paciente fue, en promedio, mayor en la admisión del paciente en relación a su seguimiento.

\section{DESCRIPTORES}

Procesos de enfermería. Personal de enfermería. Diagnóstico de enfermería. Carga de trabajo.

\footnotetext{
${ }^{1}$ Graduanda de Enfermagem da Escola de Enfermagem, Universidade de São Paulo (EEUSP). São Paulo, SP, Brasil. Bolsista PIBICCNPq. pri_o_rezende@yahoo.com.br 2 Enfermeira. Professora Livre Docente da Escola de Enfermagem, Universidade de São Paulo (EEUSP). Diretora do Departamento de Enfermagem do Hospital Universitário, Universidade de São Paulo (HU-USP). São Paulo, SP, Brasil. raqui@usp.br
} 


\section{INTRODUÇÃO}

Este estudo tem por finalidade medir o tempo despendido pela enfermeira na avaliação e registro do processo de enfermagem, após a implementação da linguagem padronizada na prática clínica. É um subprojeto da pesquisa temática Impacto de sistema padronizado de linguagem na prática clínica do enfermeiro ${ }^{(1)}$ que tem como objetivo principal avaliar o impacto da introdução da classificação de diagnósticos de enfermagem da North American Nursing Diagnosis Association International $(\text { NANDA-I })^{(2)}$, no Hospital Universitário da Universidade de São Paulo (HU-USP).

Desde a criação do HU-USP, em 1981, as enfermeiras, em consonância com a filosofia assistencial do Departamento de Enfermagem do HU-USP, para prover os cuidados de enfermagem aos pacientes, desenvolvem o Sistema de Assistência de Enfermagem (SAE), fundamentado na Teoria das Necessidades Humanas de $\mathrm{Horta}^{(3)}$ e no conceito de Autocuidado de Orem ${ }^{(4)}$, sendo adotadas três das seis fases propostas por $\operatorname{Horta}^{(3)}$ : histórico, evolução e prescrição de enfermagem.

Na perspectiva, de aprimorar o SAE e possibilitar sua informatização, as enfermeiras do Departamento de Enfermagem do HUUSP e docentes da Escola de Enfermagem da USP, em 2001, começaram a reformular os processos envolvidos nos sistemas de documentação da assistência de enfermagem para introduzir classificações de linguagens padronizadas, para documentar os diagnósticos, intervenções e resultados de enfermagem.

Considerando que padronizar a linguagem é estabelecer um acordo sobre regras para utilização de determinados termos ${ }^{(5)}$. Desta forma, ao padronizar os termos diagnósticos, busca-se estabelecer um acordo sobre os nomes ou títulos atribuídos àquelas situações que são os focos da prática clínica da enfermagem ${ }^{(6)}$.

No início de 70, na América do Norte, as iniciativas de se construir um sistema de linguagem compartilhado pelos profissionais de enfermagem ganharam corpo, com o propósito de estabelecer uma classificação internacional dos diagnósticos de enfermagem, foram realizados estudos nesta década, na medida em que vários expoentes da enfermagem reconheceram a necessidade de desenvolver uma terminologia que descrevesse os problemas de saúde tratados por enfermeiras ${ }^{(7-8)}$.

As enfermeiras do HU-USP, reconhecendo que os diagnósticos, as intervenções e os resultados de enfermagem são conteúdos que fundamentam o SAE e que esses conteúdos devem figurar nos sistemas de informação em saúde, decidiram iniciar pela implementação de classificação dos diagnósticos de enfermagem como etapa prelimi- nar que possibilita balizar as decisões sobre os resultados esperados a partir das intervenções efetuadas ${ }^{(9-10)}$.

A classificação diagnóstica possibilita que o enfermeiro vislumbre um leque mais amplo dos fenômenos que podem ser tratados pela enfermagem e que ao formular o diagnóstico, explicite a conclusão de suas interpretações e com isso focalize de forma mais clara as necessidades de cuidados o que the oferece melhores condições para estabelecer metas e selecionar intervenções potencialmente mais reais e efetivas ${ }^{(10)}$.

Diagnóstico de enfermagem, segundo a NANDA-I, é um julgamento clínico sobre as respostas, atuais ou potenciais, que indivíduos, famílias ou comunidades apresentam a problemas de saúde / processos de vida. Os diagnósticos de enfermagem provêem as bases para a seleção de intervenções de enfermagem para alcançar resultados pelos quais a enfermeira é responsável(2).

A partir de maio de 2004, após um processo de mudança planejada e participativa, todas as unidades do Departamento de Enfermagem do HU-USP introduziram, no SAE, a classificação de diagnósticos de enfermagem da NANDA-I com a finalidade de aprimorar o cuidado prestado e sua documentação na perspectiva de informatização da assistência de enfermagem.

A documentação em saúde é um aspecto que sofrerá importantes mudanças, em virtude do avanço da informatização nessa área. No Brasil, observa-se intenso movimento em direção à adoção de sistemas eletrônicos para as diversas atividades administrativas que dão suporte aos processos de trabalho assistenciais. A informatização da documentação dos processos assistenciais propriamente ditos exigirá profissionais de saúde preparados para direcionar e acompanhar mudanças que permitam alcançar resultados positivos para os processos de trabalho e também para a saúde das pessoas assistidas ${ }^{(1)}$.

Através do prontuário eletrônico, muitos benefícios podem ser obtidos como, o acesso rápido aos problemas de saúde e intervenções atuais; acesso a conhecimento científico atualizado com conseqüente melhoria do processo de tomada de decisão; melhoria de efetividade do cuidado, o que por certo contribuirá para obtenção de melhores resultados dos tratamentos realizados e atendimento aos pacientes; possível redução de custos, com otimização dos recursos ${ }^{(11)}$.

A introdução de máquinas novas, equipamentos, as unidades especializadas do cuidado e os dispositivos de monitorização eletrônicos têm mudado como as enfermeiras estão executando o cuidado. Uma das vantagens da documentação eletrônica é a melhoria na qualidade da documentação e a satisfação do usuário, além de esta poder ser feita ao lado do paciente, esta tecnologia pode 
ajudar as enfermeiras a organizar as informações e meIhorar o fluxo de trabalho ${ }^{(12)}$.

Como etapa preliminar à informatização para implementar os diagnósticos de enfermagem na prática clínica diária, os enfermeiros do HU-USP, elaboraram impressos específicos, de acordo com os diagnósticos mais freqüentes, de cada unidade, padronizaram a linguagem das prescrições de enfermagem e alteraram o registro da evolução dos resultados da assistência. O SAE passou a compreender as fases: histórico, diagnóstico, prescrição e evolução de enfermagem.

Os dados obtidos por meio do histórico de enfermagem são sintetizados e documentados no instrumento Registro de Admissão/Transferência /Alta Hospitalar. O diagnóstico, a evolução e a prescrição de enfermagem estão integrados em um único instrumento. Por conter impressos os diagnósticos de enfermagem mais freqüentes, as características definidoras, os fatores relacionados e os fatores de risco, específicos do perfil dos pacientes de cada unidade, além das atividades de enfermagem (principais) correspondentes, este instrumento foi denominado Diagnóstico-Evolução-Prescrição de Enfermagem. A evolução de enfermagem é registrada por meio das siglas em correspondência aos diagnósticos: Me (Melhorado); Pi (Piorado); I (Inalterado); R (Resolvido).

Freqüentemente, as enfermeiras do HU-USP, referiamse ao SAE como uma atividade burocrática que tomava muito tempo e as afastava do cuidado e da equipe de enfermagem. Acredita-se que o aprimoramento do processo pelo modelo proposto, com a utilização de sistemas de classificação, justamente porque padronizam linguagens, poderá favorecer a otimização da carga de trabalho, permitindo entre outros benefícios que a enfermeira utilize melhor o seu tempo para as relações humanas do cuidado. Assim, estar junto do paciente ou da família, participando e compartilhando de suas preocupações e ansiedades, permitirá o reconhecimento de novas necessidades assistenciais.

Um estudo, desenvolvido sobre o registro da documentação de dados da enfermagem, mostrou que houve um menor gasto de tempo na documentação realizada no computador quando comparado ao tempo despendido com a documentação realizada no papel, concluindo que com a documentação computadorizada houve menor tempo gasto com a documentação e maior tempo para o cuidado direto do paciente, além de fornecer maior eficiência e exatidão na documentação(13).

O processo de implementação do diagnóstico de enfermagem representou para as enfermeiras do HU-USP a expectativa de diminuição do tempo despendido na documentação do SAE e, conseqüentemente, a sua aproximação junto aos pacientes e com a equipe de enfermagem ${ }^{(9)}$.

O tempo tem se constituído em um dos recursos fundamentais de uma organização e sua gestão contribui para a melhoria nos desempenhos individual e coletivo, conseqüentemente, na produtividade.

A partir de 1881, com os trabalhos do engenheiro Frederick Taylor, em busca de uma produtividade justa e adequada de seus trabalhadores, teve início um estudo sobre o tempo despendido pelos trabalhadores na execução de suas tarefas ${ }^{(14)}$.

Taylor direcionou seus estudos para o conteúdo do tempo de trabalho e aos ritmos nos quais ele acontece, colocando como um dos pontos centrais da administração, denominada de administração científica, a introdução do cronômetro e a divisão do trabalho em fases ou elementos do trabalho, que medidos separadamente servem como base para determinar o tempo mínimo ou tempo padrão de uma atividade ${ }^{(14)}$.

Tempos medidos (cronometrados) é o método que consiste na cronometragem da operação executada pelos trabalhadores. Esse método sofreu inúmeras modificações, desde que foi estruturado por Taylor na administração científica, mas continua sendo o método mais utilizado pelas indústrias para medir trabalho. Esse método objetiva a determinação do padrão da mão-de-obra(15).

No entanto, o tempo de trabalho não possui apenas, a dimensão quantitativa, contempla também, uma dimensão qualitativa antropológica, de como os trabalhadores organizam o seu tempo nas instituições em que trabalham ${ }^{(16)}$.

A sociologia industrial concebeu o tempo como algo objetivo, mensurável, raro e precioso. Tal concepção, que se pretendeu racional, enfatizou a homogeneidade do tempo, seu caráter quantificável e uniforme. Entretanto, ao concentrar a atenção apenas sobre o tempo quantitativo, a sociologia industrial subestimou a importância do tempo qualitativo, negligenciando que o tempo é vivido como uma abstração subjetiva e heterogênea, de contornos variáveis. Assim, as relações complexas que unem os sistemas de produção, a mão-de-obra e o ambiente, originam tipos de temporalidade e ritmos temporais extremamente diversificados ${ }^{(17-18)}$.

Nessa abordagem, pretende-se estudar o efeito da mudança do modelo SAE no tempo despendido pela enfermeira, na realização deste, após a introdução de classificação de diagnósticos e a forma de sua documentação.

\section{HIPÓTESES}

\section{O presente estudo tem por hipóteses que:}

- O tempo despendido no levantamento dos dados, avaliação e prescrição de enfermagem, estimado pela enfermeira, é diferente do obtido por observação direta (dimensão qualitativa);

- A introdução de sistema padronizado de linguagem de diagnósticos de enfermagem e alteração na forma de seu 
registro mostrará diferença no tempo despendido, na realização do SAE na admissão e no seguimento de um paciente (dimensão quantitativa).

\section{OBJETIVOS}

- Medir, após a introdução de sistema padronizado de linguagem de diagnósticos de enfermagem, o tempo obtido por observação direta despendido em: levantamento e interpretação dos dados do paciente e documentação da evolução e prescrição de enfermagem.

- Identificar, após a introdução de sistema padronizado de linguagem de diagnósticos de enfermagem, a estimativa que as enfermeiras fazem quanto ao tempo despendido em: levantamento e interpretação dos dados do paciente; documentação do SAE.

- Analisar, após a introdução de sistema padronizado de linguagem de diagnósticos de enfermagem, as diferenças entre o tempo observado (dimensão quantitativa) e o tempo estimado (dimensão qualitativa) pelas enfermeiras.

\section{MÉTODO}

Trata-se de estudo exploratório descritivo prospectivo.

\section{Local}

Este projeto de pesquisa foi realizado, nas unidades do HU-USP, que possibilitaram obter as informações antes e após a implementação do diagnóstico de enfermagem, pois em algumas unidades o processo de mudança já estava sendo implementado, na época da coleta dos dados da pesquisa na fase "antes".

O HU-USP é um hospital de média complexidade, destinado ao ensino, à pesquisa e à extensão de serviços à comunidade, com base no perfil epidemiológico do Distrito de Saúde do Butantã.

Conta com 247 leitos ativados, assim distribuídos: Clínica Médica (44); Clínica Cirúrgica (44); Clínica Pediátrica (36); Clínica Obstétrica (53, sendo 48 de Alojamento Conjunto e 05 de Ginecologia); Berçário (24); UTI Adulto (20); UTI Infantil (16) e Hospital Dia (10). Possui também unidades de Ambulatório; Pronto Socorro Adulto e Pronto Socorro Infantil; Centro Cirúrgico; Central de Material e Esterilização; Centro Obstétrico; Métodos Gráficos, Endoscopia e Radiologia; Hemodiálise; e Programa de Assistência Domiciliar.

O quadro de enfermeiras assistenciais, das referidas unidades, conta com: Clínica Médica (13); Clínica Cirúrgica (12); Pediatria (13); Clínica Obstétrica (12); Berçário (9); UTI Adulto (21); UTI Infantil (14) e Hospital Dia (2); Ambulatório (6); Pronto Socorro Adulto (15); Pronto Socorro Infantil (7); Centro Cirúrgico (12); Central de Mate- rial e Esterilização (2); Centro Obstétrico (12); Métodos Gráficos, Endoscopia e Radiologia (3); Hemodiálise (3) e Programa de Assistência Domiciliar (2).

\section{Amostra}

A amostra foi composta pelas enfermeiras que atuavam nas mesmas unidades selecionadas para o estudo na fase antes à implementação da classificação de diagnóstico de enfermagem que aceitaram participar da pesquisa e assinaram o termo de consentimento livre e esclarecido. Os dados foram coletados de um total de 42 enfermeiras, correspondendo a sete enfermeiras das seguintes unidades: Alojamento Conjunto, Berçário, Pediatria, Centro Obstétrico, UTI Adulto, UTI Infantil.

\section{Coleta dos dados}

Os dados foram coletados no período de agosto/2005 a fevereiro/2006, após a introdução e sedimentação da classificação de diagnóstico de enfermagem, nas unidades do HU-USP.

\section{Instrumento}

Para a coleta de dados foi utilizado como instrumento dois formulários, um sobre o tempo estimado pela enfermeira e outro para a observação direta do tempo despendido, na realização do SAE. O formulário sobre o tempo estimado continha uma listagem com as atividades do processo de enfermagem tanto na admissão do paciente como em seu seguimento. Este formulário era preenchido pelas enfermeiras, sendo estas previamente orientadas.

O formulário para a observação direta do tempo despendido continha itens norteadores para o registro do tempo observado em cada atividade do processo de enfermagem na admissão e no seguimento de um paciente.

\section{Procedimento de coleta de dados}

Tempo estimado

Em cada unidade estudada foi entregue às enfermeiras que compuseram a amostra o formulário para o registro dos dados relativos: à caracterização das enfermeiras; ao tipo de contato com o tema diagnóstico de enfermagem e ao tempo estimado, por elas, nas atividades que compreendem: 1) levantamento e interpretação dos dados do paciente e 2) documentação do SAE nos momentos da admissão e do seguimento de um paciente.

O conjunto de atividades levantamento e interpretação dos dados do paciente é definido como quaisquer ações da enfermeira, relativas à busca de informações clínicas sobre um paciente, com a finalidade de planejar a assistência de enfermagem. Podem incluir: entrevista e exame físico do paciente, leitura de prontuário, entrevista com familiares ou acompanhantes, e consulta a outros profissionais. 
O conjunto de atividades documentação do diagnósti$c o$, evolução e prescrição de enfermagem é definido como ações de registro de sínteses do levantamento e interpretação dos dados do paciente e de registro dos cuidados planejados para o período. Podem incluir: registro no impresso de diagnósticos, registro na evolução de enfermagem e registro da prescrição de enfermagem.

\section{Tempo observado}

Em cada unidade, o tempo despendido pelas mesmas enfermeiras, foi medido, pela pesquisadora, através observação direta com o auxílio de um cronômetro, enquanto realizavam os dois conjuntos de atividades, no momento da admissão e do seguimento do paciente.

Uma unidade de observação inclui os dois conjuntos de atividades descritos. Às enfermeiras em observação foi solicitado para que realizassem os dois conjuntos de atividades para um paciente de cada vez. Com isso pretendeu-se evitar que vários pacientes fossem avaliados antes das atividades de documentação.

A unidade de observação teve início no momento em que a enfermeira, previamente orientada, informou que começaria o levantamento dos dados para o planejamento diário da assistência de um determinado paciente. Da mesma forma, o término da observação ocorreu no momento em que a enfermeira informou que concluiu todos os registros envolvidos no planejamento diário da assistência de enfermagem. $O$ tempo consumido em atividades não pertencentes às consideradas no estudo não foram mensurados.

Os tempos dos dois conjuntos de atividades foram computados com cronômetro (minutos e segundos) e registrados em formulário padronizado, precedido de ficha para registro dos dados de identificação da enfermeira e de outras variáveis de caracterização da situação observada. Cada enfermeira foi observada, em média cinco vezes, realizando as atividades do processo de enfermagem nos momentos de admissão e de seguimento de um paciente

\section{Análise dos dados}

As variáveis principais do estudo são:

- Tempo estimado para a atividade A - estimativa pela enfermeira do tempo despendido em levantamento e interpretação dos dados do paciente, indicado em minutos;

- Tempo estimado para a atividade B - estimativa pela enfermeira do tempo despendido em documentação do registro de admissão; diagnóstico; evolução e prescrição de enfermagem, indicado em minutos.

- Tempo observado para a atividade A - o tempo obtido por observação direta despendido em levantamento e interpretação dos dados do paciente, descrito em termos de média dos tempos das observações de cada enfermeira, em minutos.
- Tempo observado para a atividade B - o tempo obtido por observação direta despendido em documentação do registro de admissão; diagnóstico; evolução e prescrição de enfermagem, descrito em termos de média dos tempos das observações de cada enfermeira, em minutos.

Os dados obtidos foram lançados em planilhas eletrônicas, onde foram calculadas estatísticas para possibilitar a análise e descrição dos resultados.

\section{Aspectos éticos}

O presente estudo é um subprojeto da pesquisa temática Impacto de sistema padronizado de linguagem na prática clínica do enfermeiro (Registro CEP n. 412/03), aprovada, pela Câmara de Pesquisa e pelo Comitê de Ética em Pesquisa do HU-USP, desde 20/02/2004.

\section{APRESENTAÇ̃̃O E DISCUSSÃO DOS RESULTADOS}

\section{Caracterização da Amostra}

Neste levantamento houve a participação de 42 enfermeiras, apenas $2,4 \%$ desse grupo eram do sexo masculino. A média de idade foi de 36 anos. 0 tempo médio de anos de formada foi de 12 anos e o tempo médio de exercício da profissão foi de 11 anos. Das enfermeiras participantes, $78,6 \%$ referiram ter uma única jornada de trabaIho e $14,3 \%$ terem uma jornada dupla de trabalho.

A participação na assistência como atividade predominante foi referida por $100 \%$ das enfermeiras participantes e 33,3\% também referiram exercer função administrativa.

Das 42 enfermeiras, $40,5 \%$ trabalhavam no turno da manhã; $28,6 \%$ no turno da tarde e $30,9 \%$ no turno noturno, sendo este correspondido por turnos noturnos pares e ímpares.

A Tabela 1 apresenta as diversas modalidades de contato, das enfermeiras dessa amostra, na fase após implementação do diagnóstico de enfermagem no HU-USP com o tema diagnóstico de enfermagem. Ficou evidente que nessa fase, praticamente a maioria $(92,8 \%)$ das enfermeiras referiram vivenciar muito o tema diagnóstico de enfermagem na prática clínica; $50 \%$ também referiram fazer muita leitura do referido tema. No entanto, chama atenção que na fase após implementação da classificação de diagnóstico de enfermagem, haja, ainda, uma percentagem de $40,9 \%$ de enfermeiras que referiram pouco contato com esse tema. 
Tabela 1 - Contato dos enfermeiros com o tema "diagnóstico de enfermagem”, fase após implementação do diagnóstico de enfermagem, período de agosto/2005 a fevereiro/2006 - HU - USP - São Paulo - 2006

\begin{tabular}{|c|c|c|c|c|c|c|c|c|}
\hline Atividade & Muito & $\%$ & Pouco & $\%$ & $\begin{array}{c}\text { Quase } \\
\text { Nada }\end{array}$ & $\%$ & Nada & $\%$ \\
\hline Leitura & 21 & 50 & 20 & 47,6 & 1 & 2,4 & 0 & 0 \\
\hline Aulas & 13 & 30,9 & 28 & 66,7 & 1 & 2,4 & 0 & 0 \\
\hline Eventos & 4 & 9,5 & 24 & 57,2 & 10 & 23,8 & 4 & 9,5 \\
\hline Prática Clínica & 39 & 92,8 & 3 & 7,2 & 0 & 0 & 0 & 0 \\
\hline Pesquisa & 3 & 7,2 & 11 & 26,2 & 14 & 33,3 & 14 & 33,3 \\
\hline TOTAL & 80 & 38,1 & 86 & 40,9 & 26 & 12,0 & 18 & 8,6 \\
\hline
\end{tabular}

$\mathrm{N}=42$ enfermeiras

\section{Apresentação dos dados relativos ao tempo estimado $e$ medido após a implementação do diagnóstico de enfermagem}

Nas tabelas, a seguir estão sintetizados os dados relativos ao tempo estimado e medido que a enfermeira despende, na fase após a implementação da classificação de diagnóstico de enfermagem, nos dois conjuntos de atividade: avaliação do paciente e documentação dos dados do paciente nas situações admissão e seguimento do paciente.

Quanto ao tempo estimado pelas enfermeiras na realização da avaliação do paciente, no momento da admissão foram colhidas informações de sete enfermeiras de cada unidade, com exceção da UTI Infantil, pois a maioria dos pacientes é proveniente de outras unidades do HU-USP, tais como: Pronto Socorro Infantil, Pediatria, Centro Obstétrico e Berçário. Nesses casos, as enfermeiras da UTI Infantil, validam a documentação feita pelas unidades de origem, acrescentando alguns dados relativos a monitorização desses pacientes, nos instrumentos do SAE próprios da UTI Infantil. Na UTI Adulto, também houve dificuldade para colher o quantitativo estipulado de sete enfermeiras por unidade, devido à situação similar quanto à procedência dos pacientes nesta unidade, no entanto, houve a possibilidade de obter os dados junto a cinco enfermeiras.
A Tabela 2 mostra que o tempo médio estimado pelas enfermeiras, em todas as unidades estudadas, para avaliação do paciente no momento da admissão que compreende o exame físico e a entrevista com o paciente e ou familiar (Histórico de Enfermagem) foi, significativamente, superior ao tempo medido. Esse fato pode indicar que as enfermeiras, como são interrompidas pelas inúmeras solicitações das equipes, familiares e alunos dos cursos de graduação, durante o procedimento de admissão do paciente, percebem que o tempo de dedicação é maior do que o tempo medido, exclusivamente no desempenho desta atividade. Chama atenção o tempo medido para a avaliação do paciente na admissão do binômio mãe-filho no Alojamento Conjunto, 1,9 minuto, provavelmente como essas pacientes são provenientes do Centro Obstétrico, portanto com o processo (SAE) já iniciado, as enfermeiras apenas complementam e validam os dados que necessita para dar prosseguimento à assistência de enfermagem.

Ressalta-se que o tempo médio medido em todas as unidades, mesmo na situação de avaliação na admissão de um paciente na unidade, foi significativamente inferior quando comparado ao tempo estimado. O maior tempo médio medido foi na Pediatria, 7,5 minutos (Tabela 2).

Tabela 2 - Tempo estimado e medido (em minutos) na avaliação do paciente, na admissão após implementação do diagnóstico de enfermagem, período de agosto/2005 a fevereiro/2006 - HU - USP - São Paulo - 2006

\begin{tabular}{|c|c|c|c|c|c|c|}
\hline \multirow{2}{*}{ Unidades } & \multicolumn{3}{|c|}{ Tempo Estimado } & \multicolumn{3}{|c|}{ Tempo Medido } \\
\hline & $\mathbf{N}=\mathbf{3 3}$ & Média & Desvio Padrão & $\mathbf{N}=\mathbf{1 3 7}$ & Média & Desvio Padrão \\
\hline Alojamento Conjunto & 7 & 9,7 & 7,3 & 35 & 1,9 & 0,9 \\
\hline Berçário & 7 & 10,1 & 7,1 & 27 & 4,1 & 7,7 \\
\hline Pediatria & 7 & 22,1 & 7,6 & 34 & 7,5 & 3,7 \\
\hline UTI Adulto & 5 & 23,0 & 6,7 & 6 & 6,4 & 4,7 \\
\hline Centro Obstétrico & 7 & 10,4 & 2,1 & 35 & 5,1 & 2,4 \\
\hline
\end{tabular}

O tempo estimado pelas enfermeiras para a documentação, ou seja, o registro de admissão, diagnósticos, prescrição e evolução do paciente, na admissão do paciente, na unidade, também foi superior ao tempo medido. Os maiores tempos médios medidos, para essa atividade, foram na UTI Adulto e na Pediatria, respectivamente, 17,4 minutos e 14,7 minutos. No Centro Obstétrico o tempo médio medido, 1,6 minutos foi o mais baixo, isso pode ser explicado pela forma de construção do instrumento, onde a enfermeira registra, praticamente, todas as informações em forma de chek list (Tabela 3). 
Tabela 3 - Tempo estimado e medido (em minutos) do registro na admissão de um paciente após implementação do diagnóstico de enfermagem, período de agosto/2005 a fevereiro/2006 - HU -USP - São Paulo - 2006

\begin{tabular}{|c|c|c|c|c|c|c|}
\hline \multirow{2}{*}{ Unidades } & \multicolumn{3}{|c|}{ Tempo Estimado } & \multicolumn{3}{|c|}{ Tempo Medido } \\
\hline & $\mathbf{N}=\mathbf{3 3}$ & Média & Desvio Padrão & $\mathrm{N}=139$ & Média & Desvio Padrão \\
\hline Alojamento Conjunto & 7 & 16,6 & 6,6 & 35 & 7,3 & 1,9 \\
\hline Berçário & 7 & 19,3 & 7,3 & 28 & 7,7 & 3,7 \\
\hline Pediatria & 7 & 19,6 & 4,5 & 35 & 14,7 & 4,4 \\
\hline UTI Adulto & 5 & 23,6 & 9,6 & 6 & 17,4 & 5,4 \\
\hline Centro Obstétrico & 7 & 11,9 & 4,0 & 35 & 1,6 & 0,7 \\
\hline
\end{tabular}

O tempo estimado na avaliação do paciente durante o seguimento foi percebido pelas enfermeiras como superior ao medido tanto no que se refere à avaliação quanto à documentação das fases do SAE, como demonstrado nas tabelas 4 e 5, respectivamente. No Centro Obstétrico não há seguimento da paciente, pois toda documentação do SAE se refere ao período de pré-parto e parto. O seguimento das puérperas é realizado no Alojamento Conjunto.

Tabela 4 - Tempo estimado e medido (em minutos) na avaliação do paciente, no seguimento, após implementação do diagnóstico de enfermagem - HU - USP - São Paulo - 2005

\begin{tabular}{|c|c|c|c|c|c|c|}
\hline \multirow{2}{*}{ Unidades } & \multicolumn{3}{|c|}{ Tempo Estimado } & \multicolumn{3}{|c|}{ Tempo Medido } \\
\hline & $\mathrm{N}=35$ & Média & Desvio Padrão & $\mathrm{N}=172$ & Média & Desvio Padrão \\
\hline Alojamento Conjunto & 7 & 7,6 & 3,1 & 33 & 3,8 & 1,2 \\
\hline Berçário & 7 & 6,9 & 3,0 & 34 & 4,0 & 1,5 \\
\hline Pediatria & 7 & 6,9 & 4,2 & 35 & 2,3 & 1,2 \\
\hline UTI Adulto & 7 & 7,9 & 2,7 & 35 & 4,1 & 2,1 \\
\hline UTI Infantil & 7 & 5,7 & 3,6 & 35 & 3,5 & 1,4 \\
\hline
\end{tabular}

Tabela 5 - Tempo estimado e medido (em minutos) do registro no seguimento de um paciente após implementação do diagnóstico de enfermagem - HU - USP - São Paulo - 2005

\begin{tabular}{|c|c|c|c|c|c|c|}
\hline \multirow{2}{*}{ Unidades } & \multicolumn{3}{|c|}{ Tempo Estimado } & \multicolumn{3}{|c|}{ Tempo Medido } \\
\hline & $\mathrm{N}=35$ & Média & Desvio Padrão & $N=174$ & Média & Desvio Padrão \\
\hline Alojamento Conjunto & 7 & 10,9 & 5,4 & 34 & 4,6 & 1,4 \\
\hline Berçário & 7 & 10,3 & 3,9 & 35 & 4,4 & 1,4 \\
\hline Pediatria & 7 & 12 & 5,8 & 35 & 3,9 & 1,7 \\
\hline UTI Adulto & 7 & 17 & 6,6 & 35 & 15,6 & 4,9 \\
\hline UTI Pediátrica & 7 & 20,4 & 13,4 & 35 & 13,1 & 3,5 \\
\hline
\end{tabular}

As Tabelas 2, 3, 4 e 5 mostraram que o tempo médio estimado pelas enfermeiras foi sempre superior ao tempo médio medido. Esses dados apontam que as atividades analisadas precisam ter seus processos estudados com o intuito de otimizar a distribuição do tempo das enfermeiras em suas atividades assistenciais.

\section{CONCLUSÃO}

Através deste estudo foi possível verificar que praticamente a maioria $(92,8 \%)$ das enfermeiras referiu vivenciar muito o tema diagnóstico de enfermagem na prática clínica; $50 \%$ também referiram fazer muita leitura do referido tema.

O tempo despendido pela enfermeira na realização do SAE após a implementação do sistema padronizado de linguagem pode contribuir para que haja um maior tempo para o cuidado direto do paciente e desta forma proporcionar uma assistência de enfermagem com maior qualidade.

Um dos resultados evidenciados foi que o tempo estimado pelas enfermeiras tanto na avaliação como no registro de um paciente, tanto na admissão como no seguimento foi significativamente superior ao tempo medido, que pode ser explicado pelo fato de que o tempo percebido pelas enfermeiras reflete a realização de outras atividades realizadas concomitantemente ao SAE. O tempo médio estimado mostrou que na admissão o maior tempo médio foi na UTI Adulto 46,6 minutos, seguido pela Pediatria 41,7 minutos, Berçário 29,4, Alojamento Conjunto 26,3 e Centro Obstétrico 22,3 minutos. 
Entretanto, para o tempo médio estimado no seguimento, verificou-se que o maior tempo foi na UTI Infantil 26,1 minutos, seguido pela UTI Adulto 24,9 minutos, Pediatria 18,9 minutos, Alojamento Conjunto 18,5 minutos e Berçário 17,2 minutos.

Outro resultado evidenciado no estudo foi que o tempo despendido com a avaliação de um paciente foi em média maior na admissão em relação ao seguimento, provavelmente devido ao desconhecimento do paciente e de seu quadro clínico, sendo necessário despender um maior tempo para a realização do exame físico e da entrevista com paciente/familiar. No entanto, chama atenção que para essa situação, o tempo medido demonstrou-se baixo em todas as unidades estudadas.

O tempo médio medido (cronometrado), considerando as duas atividades, avaliação e registro, para aplicação do SAE mostrou que na admissão do paciente o maior

\section{REFERÊNCIAS}

1. Gaidzinski RR, Cruz DALM, Pimenta CAM, Soares AVN, Lima AFC, Sancinetti TR, et al. Impacto da classificação do diagnóstico de enfermagem na prática clínica do enfermeiro. In: Gaidzinski RR, Soares AVN, Lima AFC, Gutierrez BAO, Cruz DALM, Rogenski NMB, et al. Diagnóstico de enfermagem na prática clínica. Porto Alegre: Artmed; 2008. p. 354-68.

2. North American Nursing Diagnosis Association (NANDA). Diagnósticos de enfermagem da NANDA: definições e classificação 2001-2002. Porto Alegre: Artmed; 2002.

3. Horta WA. O processo de enfermagem. São Paulo: EPU; 1979.

4. Orem D. Nursing concepts of practice. New York: McGrawHill; 1979.

5. Melleiro MM, Fugulim FMT, Rogenski NMB, Gonçalves VLM, Tronchin DMR. A evolução do sistema de assistência de enfermagem no Hospital Universitário da Universidade de São Paulo: uma história de 20 anos. In: Cianciarullo TI, Gualda DMR, Melleiro MM, Anabuki MH. Sistema de Assistência de Enfermagem: evolução e tendências. São Paulo: Ícone; 2001. p. 109 - 30.

6. Cruz DALM. Fenômenos de enfermagem: desatando nós conceituais. In: Garcia TR, Nóbrega MML, organizadoras. Sistemas de classificação da prática de enfermagem: um trabalho coletivo. João Pessoa: ABEn/Idéia; 2000. p. 28-36.

7. Cruz DALM. A inserção do diagnóstico de enfermagem no processo assistencial. In: Cianciarullo TI, Gualda DMR, Melleiro MM, Anabuki MH. Sistema de Assistência de Enfermagem: evolução e tendências. São Paulo: Ícone; 2001. p. 63-84.

8. Azevedo SL. Diagnósticos de enfermagem: orientadores do cuidado ao cliente diabético. [dissertação]. Rio de Janeiro: Escola de Enfermagem Anna Nery, Universidade Federal do Rio de Janeiro; 2001. tempo médio consumido foi na unidade de UTI Adulto 23,8 minutos, seguido da Pediatria 22,2 minutos, Berçário 11,8 minutos, Alojamento Conjunto 9,2 minutos e Centro Obstétrico 6,7 minutos.

No que se refere ao tempo médio medido (cronometrado), para as duas atividades, no seguimento do paciente na unidade, verificou-se que o maior tempo médio observado foi na unidade de UTI Adulto 19,7 minutos, seguido da UTI Infantil 16,6 minutos, Berçário e Alojamento Conjunto 8,4 minutos e Pediatria 6,2 minutos.

Os dados obtidos, nesta pesquisa, possibilitarão verificar o impacto da mudança do SAE no HU-USP, com a introdução de linguagens padronizadas, neste processo, em futuras pesquisas, ao comparar os dados obtidos com os da fase antes da implementação do diagnóstico de enfermagem na prática clinica da enfermeira.

9. Carpenito LJ. Diagnósticos de enfermagem: aplicação à prática clínica. 8a ed. Porto Alegre: Artmed; 2002. Desenvolvimento dos diagnósticos de enfermagem; p. 29-34.

10. Fontes $\mathrm{CMB}$. Perfis de diagnósticos de enfermagem antes e após a implementação da classificação da NANDA-I [tese]. São Paulo: Escola de Enfermagem, Universidade de São Paulo; 2006.

11. Lima AFC. O processo de implementação do diagnóstico de enfermagem no Hospital Universitário da Universidade de São Paulo. Rev Esc Enferm USP. 2006; 40(1):111-6.

12. Massad E, Marin HF, Azevedo Neto RS, editores. O prontuário eletrônico na assistência, informação e conhecimento médico. São Paulo: H. de F. Marin; 2003.

13. Langowski $C$. The times they are a changing: effects of online nursing documentation systems. Qual Manag Health Care. 2005;14(2):121-5.

14. Korst LM, Angeja-Eusebio AC, Aydin CE, Gregory KD. Nursing documentation time during implementation of an eletronic medical record. J Nurs Adm. 2003;33(1):24-30

15.Barnes RM. Estudo de movimentos e de tempos: projeto e medida do trabalho. 6a ed. São Paulo: Edgard Blücher; 1977. Definição e finalidades do estudo de tempos e de movimentos; p. 1-7.

16. Gaidzinski RR. Dimensionamento de pessoal de enfermagem em instituições hospitalares [tese]. São Paulo: Escola de Enfermagem, Universidade de São Paulo; 1998.

17. Hassard J. Tempo de trabalho: outra dimensão esquecida nas organizações. In: Chanlat JF, editor. O indivíduo na organização: dimensões esquecidas. São Paulo: Atlas; 1992.

18. Jones AR. Time to think: temporal considerations in nursing practice and research. J Adv Nurs. 2001;33(2):150-8. 